OPEN ACCESS

Edited by:

Michael S. Dempsey,

Boston University, United States

Reviewed by:

Robert Reeve,

The University of Melbourne, Australia

Rui Alexandre Alves,

University of Porto, Portugal

*Correspondence:

Paolo Riccardo Brustio paoloriccardo.brustio@unito.it

Specialty section:

This article was submitted to Educational Psychology, a section of the journa

Frontiers in Psychology

Received: 22 March 2019 Accepted: 15 July 2019

Published: 31 July 2019

Citation:

Rabaglietti E, De Lorenzo A and Brustio PR (2019) The Role of Working Memory on Dual-Task

Cost During Walking

Performance in Childhood.

Front. Psychol. 10:1754.

doi: 10.3389/fpsyg.2019.01754

\section{The Role of Working Memory on Dual-Task Cost During Walking Performance in Childhood}

\author{
Emanuela Rabaglietti ${ }^{1}$, Aurelia De Lorenzo ${ }^{1}$ and Paolo Riccardo Brustio ${ }^{2 *}$
}

${ }^{1}$ Department of Psychology, University of Turin, Turin, Italy, ${ }^{2}$ NeuroMuscularFunction Research Group, Department of Medical Sciences, School of Exercise and Sport Sciences, University of Turin, Turin, Italy

This study examined the effect of a secondary motor task on walking ability, whether performance differed according to age and the possible relationship between cognitive abilities, specifically working memory, and dual-task costs in children with typical development. Fifty-three female children (mean age $M=10 \pm 2$ years), were divided into two different age groups: a young (7-9 years; $n=17)$ and an older group (10-13 years; $n=36)$. First, participants performed a Walking Test (WT) without additional tasks; afterward, they performed the same walking test while performing each of the following tasks: carrying (1) a glass of water, (2) a ball on a round tray and (3) the combination of both tasks (1) and (2). The Test of Memory and Learning were used to assess working memory. WTs under a dual-task condition generally produced worse results compared to a single-task condition $[F(3,135)=32.480, p<0.001]$. No age-related difference was observed $[F(1,45)=0.497, p=0.485]$. Age, digit forward and backward, facial memory, and paired recall accounted altogether for $28.6 \%$ of variance in dual-task ability during WT while carrying a glass of water and a ball on a round tray. Specifically, facial memory significantly accounted for the variance of DTC in WTWT ( $\beta=-0.381, p=0.016)$. Moreover, a trend toward a statistical significance was observed for digit forward $(\beta=-0.275$, $p=0.085)$. Results underlined that regardless of the age, a dual-task performance might affect walking performance depending on the required secondary task. Moreover, our results showed the association between working memory skills and dual-task cost in walking ability.

Keywords: cognitive abilities, motor development, school age, dual-task activity, walking

\section{INTRODUCTION}

Working memory is an integral component of executive functions (Anderson et al., 2010), responsible for the complex cognitive coordination necessary for everyday life activity (Doherty et al., 2018). In the literature, different multiple definitions were given about working memory depending on the assumed theoretical model (e.g., multiple-component model, time-based resource sharing, and embedded process) (Cowan, 2017; Doherty et al., 2018). One of the most studied frameworks of multi-component system includes the model of Baddeley and Hitch (1974). According to this view, working memory includes four separable but interacting subsystems (Baddeley, 2000, 2012): (1) a supervisory system controlling and regulating the 
cognitive processes, named central executive, that coordinates separate executive functions such as inhibition, task-switching, and dual-tasking (Doherty et al., 2018). Two slave systems: (2) the phonological loop which includes both verbal inputs subject to a rapid decay and a rehearsal process that can be used to refresh decaying representations (Baddeley, 1996) and (3) the visuospatial sketchpad which processes and maintains visual and spatial material (Baddeley and Lieberman, 1980). Finally, (4) a multidimensional passive storage system, which links information across the two slave systems, the long-term memory storage and the perception, named episodic buffer (Baddeley, 2012). We frame our study within the multi-component working memory model.

Working memory capacity linearly develops across childhood and reaches the adult level during adolescence (Gathercole, 1998; Bathelt et al., 2017), depending on brain structure maturations (Bathelt et al., 2017). The phonological loop appears to be intact in children but, only after age 7 , the child increases the amount of verbal material and is able to maintain and rehearse spontaneously (Gathercole et al., 2004). Developmental data for visuospatial sketchpad support the division between visual and spatial components and show an increase in both components as age increases (Swanson, 1999; Mammarella et al., 2008) but with different rates and trajectories (Hamilton et al., 2003).

Working memory conflicts between tasks are a major source of interference in dual tasking (Nijboer et al., 2016). Indeed, dual-task methodology is used to distinguish developmental changes in the different components of working memory (Willis and Gathercole, 2001; Conway et al., 2005; Jaroslawska et al., 2018). Specifically, dual-task methodology requires simultaneous processing and storage demands, such as reading while increasing the series of allowed sentences and eventually recalling the last word of each sentence (Daneman and Carpenter, 1980). In this situation, the simultaneous performances, that rely on the same component of working memory, lead to a competition in cognitive resources with a reduction in working memory capacity (i.e., decrease of the amount of additional information that can be maintained) and less efficient performance when two tasks are separately performed (Daneman and Carpenter, 1980; Jaroslawska et al., 2018). On the contrary, when the performance of two tasks requires the use of two domainspecific slave systems (i.e., use of verbal and visuospatial information) the performance of the two tasks is as efficient as the performance of a single task (Thomason et al., 2009).

Similar to working memory, the development of walking skills occurs according to different processes from the first years of life until adolescence (Shumway-Cook and Woollacott, 2012). In the first years of life, walking is characterized by rapid improvements in gait pattern (Kraan et al., 2017). Mature and independent walking is reached around age 2-3 years. After this, a child shows a refinement of gait pattern, such a reduction in the base of support width, a clear toe-off and heal strikes and reciprocal arm swing (for a review, please consult Kraan et al., 2017), until walking becomes steady and similar to the adult pattern around age 7-8 years (Sutherland, 1997; Kraan et al., 2017). Nevertheless, walking patterns can be refined and improved after this age (Belmonti et al., 2013;
Manicolo et al., 2016), also due to the maturation in brain structure (Leisman et al., 2016).

During everyday life activities, single-task motor performances are rarely carried out alone, instead dual-task performances are more common. These situations, including dual-task activities, may lead to a change in one of the two activities and may be particularly challenging during childhood. Indeed, the simultaneous performance of a secondary task, such as a motor or cognitive task or both, together with a primary task, may affect the primary task, such as the walking task (Huang et al., 2003; Ruffieux et al., 2015; Saxena et al., 2017; Brustio et al., 2017b; Schott and Klotzbier, 2018). Attentional resources are limited (Kahneman, 1973; Navon and Gopher, 1979) when shared between the primary and secondary tasks (Wickens, 1991). Consequently, due to a competition of the same pool of cognitive resources (Navon and Gopher, 1979), a worsening of the performance occurs, known as dual-task cost (e.g., there is a reduction of performance in dual-task performance, compared to single-task performance).

Studies on walking tasks underlined a decrease in performance both with additional motor tasks (Huang et al., 2003; Cherng et al., 2007; Hung et al., 2013; Abbruzzese et al., 2014) and cognitive tasks (Schaefer et al., 2015; Schott and Klotzbier, 2018). For example, Cherng et al. (2007) investigated the impact of concurrent motor and cognitive tasks on walking ability in preschool children (age 4-6 years) and showed that the concurrent performance of a secondary task negatively affected the gait parameters, depending on the difficulty and type of the secondary task. However, some studies showed an improvement of dualtask performance with children's growth, while other studies did not confirm this evidence (for a further review on the topic, see: Saxena et al., 2017). For example, Boonyong et al. (2012) underlined that postural control while performing a walking task under dual-task condition improves as age increases. Differently, Hagmann-von Arx et al. (2016) did not report the effect of age on walking ability with an additional cognitive task. We can assume that the variation in the use of non-standardized test protocols (e.g., different additional tasks) may have affected these results. Despite the above findings, more attentional resources during childhood development are required for walking control (Boonyong et al., 2012; Chauvel et al., 2017).

Walking is one of the most common human activities. However, under dual-task conditions, it is a cognitively demanding activity (Yogev-Seligmann et al., 2008). Different studies investigated the relationship between cognitive resources and walking abilities under dual-task conditions particularly in older people (YogevSeligmann et al., 2008), but, to our knowledge, studies on childhood are limited. Using an additional cognitive task during walking performance, Hagmann-von Arx et al. (2016) did not find any association between the ability to walk during dual-task condition and cognitive abilities in childhood. However, there is a gap in the literature regarding the relationship between the cognitive function and walking ability under the dual-task performance with a secondary motor task. Thus, we investigated (1) the effect of a secondary motor task on walking ability, (2) whether performance differed according to age, and (3) the possible relationship between cognitive abilities, specifically working 
memory, and dual-task costs during children's typical development. In particular, using the framework of multi-component systems, we wanted to investigate the role, if any, of working memory on walking ability under the dual-task condition. Indeed, given that task-relevant information is controlled, regulated, and actively maintained by working memory (Beurskens and Bock, 2012), it is plausible to speculate that it may be associated with walking ability under the dual-task condition.

\section{MATERIAL AND PROCEDURE}

\section{Participants}

Fifty-three female children with typical development, aged $7-13$ years (mean age $M=10 \pm 2$ years), participated in the study. Children were divided into two different age groups: a young (7-9 years; $n=17$ ) and an older group (10-13 years; $n=36$ ). All participants were naive to the purpose of the study; they had normal or corrected-to-normal eye sight, normal hearing and no neuromuscular, neurological, or cardiovascular diseases nor attentional deficits, according to their parents' reports. The study was conducted in conformity with the recommendations of the Ethics Committee of the University of Torino (Protocol Number: N. 60193). Before starting the study, each parent or legal guardian read, concurred, and signed a written informed consent in accordance with the Declaration of Helsinki.

\section{Measurements}

The Italian version of Test of Memory and Learning (TOMAL; Reynolds and Bigler, 1994; Reynolds et al., 1995) was used to assess cognitive abilities (i.e., memory and learning). The TOMAL is a validated battery designed to assess memory functions (i.e., associative and free recall, verbal memory, attention, and concentration) in children and adolescents, from 5 to 19 years, 11 months and 30 days. TOMAL is composed of 10 core subtests divided into verbal and non-verbal indexes (i.e., five subtests for each index) and four optional supplemental subtests. The combination of these two indexes produces the Composite Memory Index. Internal consistency reliability for each subtest ranges from 0.75 to 0.99 (Cronbach Alpha coefficients) and test-retest reliability ranges from 0.71 to 0.92 . For more details about TOMAL subtests see Reynolds and Bigler (1994). The following subtests were used for this study:

1. digit forward: this verbal subtest measures rote learning, sequential memory, and attention and concentration and requires the capability to recall a list of numbers in the correct order. The score was the total number of items correctly repeated. The Cronbach alpha coefficient for ages $7-13$ years is 0.97 .

2. digit backward: this verbal subtest measures attention, concentration, and the backward span tasks which tap into working memory and requires the capability to recall a list of numbers in reverse order. The score was the total number of items correctly repeated. The Cronbach alpha coefficients for ages 7-13 years of this subtest range from 0.96 to 0.98 .
3. facial memory: this subtest measures visual and deferring memory and requires capability to recognize faces from different ethnicities, gender, and age in black and white photos. The score was the total number of recognized photos. The Cronbach alpha coefficients for ages 7-13 years of this subtest range from 0.75 to 0.8 .

4. paired recall: this subtest measures learning and associative recall and requires the capability to pair a prompted word with an associated word. The score was the total number of paired words. The Cronbach alpha coefficients for ages $7-13$ years of this subtest range from 0.83 to 0.91 .

Physical measures included a single-task trial and three different dual-task trials. The single-task trials consisted in the performance of a Walking Test (WT) along a pathway of 14 meters. The participants were instructed to walk at their self-selected speed. The walking speed was measured using a digital stopwatch to the nearest $0.01 \mathrm{~s}$. The first and the last meter were not included in the analysis and considered as acceleration and deceleration phases. Each child completed WT while performing. The following additional secondary task (Brustio et al., 2018a,c):

1. carrying a glass of water (filled to $10 \mathrm{~mm}$ from the rim) with the preferred hand (WTW);

2. carrying a ball (weight: $100 \mathrm{~g}$, diameter: $7 \mathrm{~cm}$ ) on a round tray (weight: $50 \mathrm{~g}$, diameter: $17 \mathrm{~cm}$ ) with the dominant hand only (WTT);

3. carrying a glass of water (filled to $10 \mathrm{~mm}$ from the rim) and a ball (weight: $100 \mathrm{~g}$, diameter: $7 \mathrm{~cm}$ ) on a round tray (weight: $50 \mathrm{~g}$, diameter: $17 \mathrm{~cm}$ ) with the dominant hand only (WTWT).

Participants first performed WT with a single-task condition followed by WT with a dual-task condition. The WT with a dual-task condition was performed in a counterbalanced order. Dual-task walking conditions included one familiarization trial ahead of the test trial (Hagmann-von Arx et al., 2016). The trial was repeated if any of the above dual-task walking conditions failed (Cherng et al., 2007). No instructions were given regarding which task to prioritize (Brustio et al., 2017a).

Participants were individually tested by the same investigator and evaluated in 1 day, with a 3-min break between each cognitive subtest and walking test.

\section{Analysis}

Repeated measures of analysis of variance (ANOVA) with withinsubject factor Task Condition (Task Condition: WT, WTW, WTT, and WTWT) and between-subject factor Age Group (Age Group: young and older group) were run to determine withinsubject and age-related differences in walking performance with a dual-task condition. Significant results were followed up by means of post hoc analysis with Bonferroni correction.

Dual-task costs (DTC) were calculated as the difference between the scores of the dual-task and single-task performances in order to quantify the participants' dual-task ability (Brustio et al., 2018b). A positive value of DTC indicates 
lower dual-task ability while a negative value indicates higher dual-task ability.

Hierarchical multiple regression analyses were conducted to assess the relationship between DTCs and cognitive performance. Specifically, we used each DTC (e.g., DTC in WTW, WTT, and WTWT) as a dependent variable and age, digit forward, digit backward, facial memory, and paired recall as independent factors. Specifically, independent factors were entered in three steps of the regression model with the following order: age in Step 1; age and digit forward and backward subtests (i.e., verbal memory, attention, and concentration) in Step 2; age, digit forward and backward facial memory and paired recall subtests (i.e., associative and free recall) in Step 3.

\section{RESULTS}

The test performances are summarized in Table $\mathbf{1}$ and Figure $\mathbf{1}$. Overall, a significant difference was observed in Task Condition $\left[F(3,135)=32.480, p<0.001\right.$, partial $\left.\eta^{2}=0.419\right]$ but not for Age Group $\left[F(1,45)=0.497, p=0.485\right.$, partial $\left.\eta^{2}=0.011\right]$ and Task Condition $\times$ Age Group interaction $[F(1,45)=0.256$, $p=0.615$, partial $\left.\eta^{2}=0.006\right]$. Post hoc analysis with a Bonferroni adjustment underlined a statistically significant worse walking performance in WTW [3.95 s, 95\% CI $(2.21,5.57), p<0.001$ ], WTT $[5.28$ s, 95\% CI $(1.98,8.59), p<0.00]$, and WTWT [10.57 s, 95\% CI $(6.44,15.39), p<0.001]$ compared to WT. A significant worse walking performance was observed in WTWT compared to WTW $[6.96 \mathrm{~s}, 95 \%$ CI $(3.58,10.35)$, $p<0.001$ ] and WTT [5.63 s, 95\% CI $(3.78,8.06), p<0.001]$. No significant difference was observed between WTW and WTT $(p>0.05)$. For more details, see Figure 1A.

Table 2 presents the results of hierarchical multiple regression analyses obtained in the different steps. Independent factors were not associated with DTC in WTW $[F(5,44)=2.201, p=0.074$, $\left.R^{2}=0.220\right]$ nor in WTT $\left[F(5,44)=2.188, p=0.075, R^{2}=0.219\right]$. However, the addition of facial memory and paired recall to the prediction of DTC in WTW (Step 3) led to a statistically significant increase in $R^{2}$ of $0.120[F(1,39)=3.425, p=0.043]$.

TABLE 1 | Results of test cognitive and motor performance.

\begin{tabular}{lcc}
\hline Variables & $\boldsymbol{M}$ & SD \\
\hline Digit forward (scores) & 43.19 & 14.78 \\
Digit backward (scores) & 30.41 & 17.87 \\
Facial memory (scores) & 23.63 & 5.12 \\
Paired recall (scores) & 28.00 & 5.11 \\
WT (s) & 7.38 & 1.61 \\
WTW (s) & 11.29 & 4.73 \\
WTT (s) & 12.79 & 8.28 \\
WTWT (s) & 17.95 & 11.11 \\
DTC WTW (s) & 3.91 & 1.06 \\
DTC WTT (s) & 5.40 & 7.70 \\
DTC WTWT (s) & 10.57 & 10.48
\end{tabular}

M, mean; SD, standard deviation; WT, Walking Test; WTW, Walking Test while carrying a glass of water; WTT, Walking Test while carrying a ball on a round tray; WTWT, WT while carrying a glass of water and a ball on a round tray; DTC, dual-task cost.
Differently, independent factors accounted altogether for $28.6 \%$ of the variance in DTC in WTWT $[F(5,44)=3.130, p=0.018]$. The addition of digit forward and backward to the model (Step 2) led to an increase in $R^{2}$ of $0.136, F(2,41)=3.236, p=0.05$, while the addition of facial memory and paired recall (Step 3) also led to an increase in $R^{2}$ of $0.146, F(1,39)=3.998, p=0.026$. Specifically, facial memory significantly accounted for the variance of DTC in WTWT $(\beta=-0.381, p=0.016)$. Moreover, a trend toward a statistical significance was observed for digit forward $(\beta=-0.275, p=0.085)$.

\section{DISCUSSION}

The aims of this study were to investigate (1) the effect of a secondary motor task on walking performance, (2) whether children's performance differed according to age, and (3) the possible relationship between cognitive abilities, specifically working memory, and dual-task costs in children with typical development. For this purpose, we evaluated the concurrent performance of walking with different secondary motor tasks, i.e., while carrying a glass of water (WTW), while carrying a ball on a round tray (WTT), and while carrying a glass of water and a ball on a round tray, using the preferred hand (WTWT). Results underlined that an additional task may affect walking performance depending on the required secondary task.

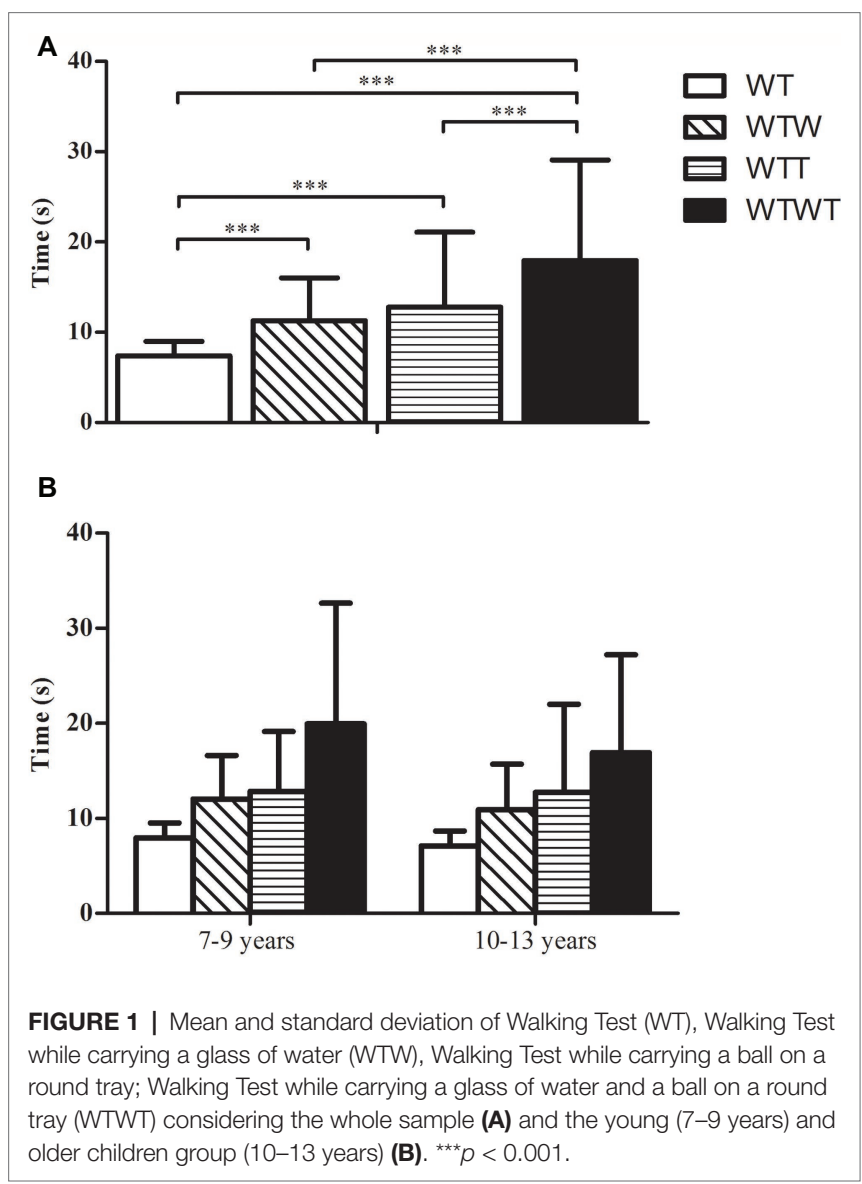


TABLE 2 | Summary of hierarchical regression analyses on dual-task costs.

\begin{tabular}{|c|c|c|c|c|c|c|}
\hline Dual-task cost & Independent variables & $R^{2}$ & $\Delta R^{2}$ & $\beta$ & Partial $r$ & $p$ \\
\hline \multicolumn{7}{|l|}{ WTW } \\
\hline Step 1 & Age & 0.001 & & -0.007 & -0.007 & 0.965 \\
\hline & Digit forward & & & -0.317 & -0.288 & 0.061 \\
\hline & Digit backward & 0.083 & 0.082 & 0.127 & 0.120 & 0.442 \\
\hline \multirow[t]{3}{*}{ Step 3} & Age & & & -0.022 & -0.023 & 0.886 \\
\hline & Facial memory & & & -0.332 & -0.319 & 0.042 \\
\hline & Paired recall & 0.220 & 0.137 & -0.198 & -0.188 & 0.240 \\
\hline \multicolumn{7}{|l|}{ WTT } \\
\hline Step 1 & Age & 0.007 & & 0.081 & 0.007 & 0.597 \\
\hline \multirow[t]{3}{*}{ Step 2} & Age & & & 0.020 & 0.044 & 0.894 \\
\hline & Digit forward & & & -0.225 & 0.288 & 0.174 \\
\hline & Digit backward & 0.108 & 0.101 & -0.161 & 0.020 & 0.325 \\
\hline Step 3 & Paired recall & 0.219 & 0.111 & -0.250 & 0.020 & 0.140 \\
\hline \multicolumn{7}{|l|}{ WTWT } \\
\hline Step 1 & Age & 0.004 & & -0.066 & -0.066 & 0.665 \\
\hline \multirow{3}{*}{ Step 2} & Age & & & -0.126 & -0.132 & 0.399 \\
\hline & Digit forward & & & -0.392 & -0.358 & 0.018 \\
\hline & Digit backward & 0.140 & 0.136 & 0.055 & 0.054 & 0.729 \\
\hline \multirow[t]{5}{*}{ Step 3} & Age & & & -0.086 & -0.095 & 0.553 \\
\hline & Digit forward & & & -0.275 & -0.272 & 0.085 \\
\hline & Digit backward & & & 0.199 & 0.196 & 0.219 \\
\hline & Facial memory & & & -0.381 & -0.375 & 0.016 \\
\hline & Paired recall & 0.286 & 0.146 & -0.134 & -0.134 & 0.402 \\
\hline
\end{tabular}

WTW, walking test while carrying a glass of water; WTT, walking test while carrying a ball on a round tray; WTWT, walking test while carrying a glass of water and a ball on a round tray.

Moreover, no age-related differences in dual-task performance were observed. Finally, our results showed the association between facial memory skills and walking ability in dual-task performance while carrying a glass of water and a ball on a round tray.

\section{Walking Performance During Dual-Task}

Considering the primary aim of the study, our results found that dual-task walking performances were generally worse when compared to a single-task condition (see Figure 1), showing a significant effect on the additional motor task. The observed changes may be attributed to the limited cognitive resources in children's information-processing capability (Granacher et al., 2011; Hung et al., 2013). Because of the development process in postural control ability, the walking and dynamic balance performances are impaired (Granacher et al., 2011). Consequently, children require an increase in cognitive resources to maintain the postural control under dual-task conditions (Ruffieux et al., 2015). Interestingly, WTWT was higher compared to WTW and WTT, suggesting that walking performance under a dualtask depends on the level of difficulty (Patel et al., 2014; Brustio et al., 2017b; Schott and Klotzbier, 2018).

\section{Age-Related Difference in Walking Performance During Dual-Task}

Our results suggested a similar trend in dual-task performance among young and older groups. In other words, we found that, independently from the additional required motor task, both young and older children manage their walking performance similarly. Using a walking task with additional motor tasks (i.e., carrying a box) Hung et al. (2013) showed that younger children (4-6 years old) had less bimanual coordination, as well as greater difficulty in walking task, compared to 7-13 years old children. On the contrary, no difference was observed between a younger (7-9 years old) and an older group (10-13 years old) under dual-task constraints (Hung et al., 2013). Indeed, a weak evidence of interference in younger children (2-6 years) compared to older children (7 and older) appeared when the task conditions were difficult or complex (Saxena et al., 2017).

\section{Relationship Between Working Memory and Dual-Task Costs}

When focusing on the relationship between cognitive abilities and dual-task costs, we found a negative relation between DTC (i.e., carrying a glass of water) and visual memory and recall (i.e., facial memory subtests). It is possible to speculate that during WTW children used the non-verbal information of the visuospatial sketchpad processed by the central executive (e.g., visual information storage and processing) to manage two independent streams of visual information, one related to the walking task and the other related to the secondary task (Beurskens and Bock, 2012). 
Differently from the WTW costs, when the difficulty of the secondary task increased (i.e., carrying a glass of water and a ball on a round tray) we found a negative association with visual memory and recall and a negative trend with verbal memory, rote learning, attention, and concentration (i.e., digitforward subtest). Thus, in order to manage the two concurrent tasks, both the visuospatial sketchpad processing by the central executive and the elaboration of verbal information were involved. Indeed, according to Luria (1961) and Vygotsky (1934) when children perform complex tasks (i.e., multitasking), they activate the regulatory role of language, i.e., inner speech (Emerson and Miyake, 2003), to support more demanding executive tasks (Baddeley, 2012). Inner speech plays a role in task switching performance and is associated with the phonological loop system and supports the executive control process in Baddeley's working memory model (Emerson and Miyake, 2003). Contrary to our results, Hagmann-von Arx et al. (2016) found no relationship between spatial working memory and walking ability during dual-task performance. Their results may have been influenced by the different nature of the secondary task: either listening to and recalling digits or pressing a button as well as by the use of differently used cognitive tests.

\section{Limitations}

Our study presents some limitations. Due to the relatively small sample size of the study, we were not able to extend our conclusions to the larger children's population and to study age-related differences. Moreover, the cross-sectional nature of the study did not allow us to investigate the possible causal relationship between cognitive and physical ability. Moreover, we evaluated walking performance by means of a single parameter (time), and the secondary tasks used in the present study were motor tasks only. Another limitation to our study was that single and dual-task conditions were not counterbalanced. Thus, our participants first performed the walking performance as a single-task, followed by dual-task conditions. Therefore, potential practice or fatigue effects were not controlled. As previously suggested (Schott and Klotzbier, 2018), it is possible that counterbalancing may have increased the magnitude of the observed differences among single and dual-task performances. Finally, our primary goal was to investigate the effect of a secondary task on walking performance and therefore the secondary task was not evaluated. Thus, no data on bidirectional dual-task cost were provided.

\section{REFERENCES}

Abbruzzese, L. D., Rao, A. K., Bellows, R., Figueroa, K., Levy, J., Lim, E., et al. (2014). Effects of manual task complexity on gait parameters in school-aged children and adults. Gait Posture 40, 658-663. doi: 10.1016/j.gaitpost.2014.07.017

Anderson, V., Jacobs, R., and Anderson, P. J. (2010). Executive functions and the frontal lobes: A lifespan perspective. New York: Psychology Press Taylor \& Francis Group.

Baddeley, A. D. (1996). Exploring the central executive. Q. J. Exp. Psychol. 49, 5-28. doi: $10.1080 / 713755608$

\section{CONCLUSION}

In conclusion, our results show that regardless of age, a dualtask performance may affect walking ability, depending on the required secondary task. Moreover, our results showed an association between working memory skills and dual-task cost in children with typical development.

\section{DATA AVAILABILITY}

The datasets generated for this study are available on request to the corresponding author.

\section{ETHICS STATEMENT}

The study was conducted in conformity with the recommendations of the University of Torino Ethics Committee. Before starting the study, each parent or legal guardian read, concurred and signed a written informed consent in accordance with the Declaration of Helsinki. The Ethical Committee of the University of Torino approved the study (Protocol Number: N. 60193).

\section{AUTHOR CONTRIBUTIONS}

$\mathrm{ER}, \mathrm{AD}$, and $\mathrm{PB}$ contributed to conceptualization. $\mathrm{AD}$ and $\mathrm{PB}$ contributed to investigation. $\mathrm{PB}$ contributed to formal analysis. $\mathrm{ER}, \mathrm{AD}$, and $\mathrm{PB}$ contributed to writing - original draft. ER, $\mathrm{AD}$, and $\mathrm{PB}$ contributed to writing - review and editing. ER contributed to supervision.

\section{FUNDING}

All the funding regarding the realization of this study was received internally to the authors' organization. There was no additional external funding received for this study.

\section{ACKNOWLEDGMENTS}

The authors would like to thank Lynda Lattke for reviewing the language in the manuscript.

Baddeley, A. D. (2000). The episodic buffer: a new component of working memory? Trends Cogn. Sci. 4, 417-423. doi: 10.1016/S1364-6613(00)01538-2

Baddeley, A. (2012). Working memory: theories, models, and controversies. Annu. Rev. Psychol. 63, 1-29. doi: 10.1146/annurev-psych-120710-100422

Baddeley, A. D., and Hitch, G. (1974). "Working memory" in The psychology of learning and motivation: Advances in research and theory. Vol. 8, ed. G. H. Bower (New York: Academic Press), 47-89.

Baddeley, A. D., and Lieberman, K. (1980). "Spatial working memory" in Attention and performance, VIII. ed. R. Nickerson (Hillsdale, NJ: Lawrence Erlbaum Associates), 521-540. 
Bathelt, J., Gathercole, S. E., Johnson, A., and Astle, D. E. (2017). Differences in brain morphology and working memory capacity across childhood. Dev. Sci. 21:e12579. doi: 10.1111/desc.12579

Belmonti, V., Cioni, G., and Berthoz, A. (2013). Development of anticipatory orienting strategies and trajectory formation in goal-oriented locomotion. Exp. Brain Res. 227, 131-147. doi: 10.1007/s00221-013-3495-3

Beurskens, R., and Bock, O. (2012). Age-related deficits of dual-task walking: a review. Neural Plast. 2012:131608. doi: 10.1155/2012/131608

Boonyong, S., Siu, K. C., van Donkelaar, P., Chou, L. S., and Woollacott, M. H. (2012). Development of postural control during gait in typically developing children: the effects of dual-task conditions. Gait Posture 35, 428-434. doi: 10.1016/j.gaitpost.2011.11.002

Brustio, P. R., Liubicich, M. E., Chiabrero, M., and Rabaglietti, E. (2018a). Dancing in the golden age: a study on physical function, quality of life, and social engagement. Geriatr. Nurs. 39, 635-639. doi: 10.1016/j. gerinurse.2018.04.013

Brustio, P. R., Magistro, D., Rabaglietti, E., and Liubicich, M. E. (2017a). Agerelated differences in dual task performance: a cross-sectional study on women. Geriatr Gerontol Int 17, 315-321. doi: 10.1111/ggi.12700

Brustio, P. R., Magistro, D., Zecca, M., Liubicich, M. E., and Rabaglietti, E. (2018b). Fear of falling and activities of daily living function: mediation effect of dual-task ability. Aging Ment. Health, 22, 856-861. doi: 10.1080/ 13607863.2017.1318257

Brustio, P. R., Magistro, D., Zecca, M., Rabaglietti, E., and Liubicich, M. E. (2017b). Age-related decrements in dual-task performance: comparison of different mobility and cognitive tasks. A cross sectional study. PLoS One 12:e0181698. doi: 10.1371/journal.pone.0181698

Brustio, P. R., Rabaglietti, E., Formica, S., and Liubicich, M. E. (2018c). Dualtask training in older adults: the effect of additional motor tasks on mobility performance. Arch. Gerontol. Geriatr. 75, 119-124. doi: 10.1016/j. archger.2017.12.003

Chauvel, G., Palluel, E., Brandao, A., Barbieri, G., Nougier, V., and Olivier, I. (2017). Attentional load of walking in children aged 7-12 and in adults. Gait Posture 56, 95-99. doi: 10.1016/j.gaitpost.2017.04.034

Cherng, R.-J., Liang, L.-Y., Hwang, S., and Chen, J.-Y. (2007). The effect of a concurrent task on the walking performance of preschool children. Gait Posture 26, 231-237. doi: 10.1016/j.gaitpost.2006.09.004

Conway, A. R., Kane, M. J., Bunting, M. F., Hambrick, D. Z., Wilhelm, O., and Engle, R. W. (2005). Working memory span tasks: a methodological review and user's guide. Psychon. Bull. Rev. 12, 769-786. doi: 10.3758/ BF03196772

Cowan, N. (2017). The many faces of working memory and short-term storage. Psychon. Bull. Rev. 24, 1158-1170. doi: 10.3758/s13423-016-1191-6

Daneman, M., and Carpenter, P. A. (1980). Individual differences in working memory and reading. J. Mem. Lang. 19, 450-466. doi: 10.1093/acprof:o so/9780198528012.003.0010

Doherty, J. M., Belletier, C., Rhodes, S., Jaroslawska, A., Barrouillet, P., Camos, V., et al. (2018). Dual-task costs in working memory: an adversarial collaboration. J. Exp. Psychol. Learn. Mem. Cogn. doi: 10.1037/xlm0000668

Emerson, M. J., and Miyake, A. (2003). The role of inner speech in task switching: a dual-task investigation. J. Mem. Lang. 48, 148-168. doi: 10.1016/ S0749-596X(02)00511-9

Gathercole, S. E. (1998). The development of memory. J. Child Psychol. Psychiatry $39,3-27$.

Gathercole, S. E., Pickering, S. J., Ambridge, B., and Wearing, H. (2004). The structure of working memory from 4 to 15 years of age. Dev. Psychol. 40, 177-190. doi: 10.1037/0012-1649.40.2.177

Granacher, U., Muehlbauer, T., Gollhofer, A., Kressig, R. W., and Zahner, L. (2011). An intergenerational approach in the promotion of balance and strength for fall prevention - a mini-review. Gerontology 57, 304-315. doi: $10.1159 / 000320250$

Hagmann-von Arx, P., Manicolo, O., Lemola, S., and Grob, A. (2016). Walking in school-aged children in a dual-task paradigm is related to age but not to cognition, motor behavior, injuries, or psychosocial functioning. Front. Psychol. 7:352. doi: 10.3389/fpsyg.2016.00352

Hamilton, C., Coates, R., and Heffernan, T. (2003). What develops in visuospatial working memory development? Eur. J. Cogn. Psychol. 15, 43-69. doi: $10.1080 / 09541440303597$
Huang, H. J., Mercer, V. S., and Thorpe, D. E. (2003). Effects of different concurrent cognitive tasks on temporal-distance gait variables in children. Pediatr. Phys. Ther. 15, 105-113. doi: 10.1097/01.PEP.0000067886.96352.6B

Hung, Y. C., Meredith, G. S., and Gill, S. V. (2013). Influence of dual task constraints during walking for children. Gait Posture 38, 450-454. doi: 10.1016/j.gaitpost.2013.01.009

Jaroslawska, A. J., Gathercole, S. E., and Holmes, J. (2018). Following instructions in a dual-task paradigm: evidence for a temporary motor store in working memory. Q. J. Exp. Psychol. 71:2439-2449. doi: $10.1177 / 1747021817743492$

Kahneman, D. (1973). Attention and effort. Englewood Cliffs, NJ: Prentice Hall.

Kraan, C. M., Tan, A. H. J., and Cornish, K. M. (2017). The developmental dynamics of gait maturation with a focus on spatiotemporal measures. Gait Posture 51, 208-217. doi: 10.1016/j.gaitpost.2016.10.021

Leisman, G., Moustafa, A. A., and Shafir, T. (2016). Thinking, walking, talking: integratory motor and cognitive brain function. Front. Public Health 4:94 doi: $10.3389 /$ fpubh.2016.00094

Luria, A. R. (1961). The role of speech in the regulation of normal and abnormal behavior. Oxford, England: Liveright.

Mammarella, I. C., Pazzaglia, F., and Cornoldi, C. (2008). Evidence for different components in children's visuospatial working memory. Br. J. Dev. Psychol. 26, 337-355. doi: 10.1348/026151007X236061

Manicolo, O., Grob, A., Lemola, S., and Hagmann-von Arx, P. (2016). Agerelated decline of gait variability in children with attention-deficit/hyperactivity disorder: support for the maturational delay hypothesis in gait. Gait Posture 44, 245-249. doi: 10.1016/j.gaitpost.2015.12.012

Navon, D., and Gopher, D. (1979). On the economy of the human-processing system. Psychol. Rev. 86, 214-255. doi: 10.1037/0033-295X.86.3.214

Nijboer, M., Borst, J., van Rijn, H., and Taatgen, N. (2016). Contrasting single and multi-component working-memory systems in dual tasking. Cogn. Psychol. 86, 1-26. doi: 10.1016/j.cogpsych.2016.01.003

Patel, P., Lamar, M., and Bhatt, T. (2014). Effect of type of cognitive task and walking speed on cognitive-motor interference during dual-task walking. Neuroscience 260, 140-148. doi: 10.1016/j.neuroscience.2013.12.016

Reynolds, C. R., and Bigler, E. D. (1994). Test of memory and learning (TOMAL). Austin, TX: Pro-Ed.

Reynolds, C. R., Bigler, E. D., and Ianes, D. (1995). Test TEMA. Test di memoria e apprendimento. Trento: Erickson.

Ruffieux, J., Keller, M., Lauber, B., and Taube, W. (2015). Changes in standing and walking performance under dual-task conditions across the lifespan. Sports Med. 45, 1739-1758. doi: 10.1007/s40279-015-0369-9

Saxena, S., Cinar, E., Majnemer, A., and Gagnon, I. (2017). Does dual tasking ability change with age across childhood and adolescence? A systematic scoping review. Int. J. Dev. Neurosci. 58, 35-49. doi: 10.1016/j.ijdevneu. 2017.01.012

Schaefer, S., Jagenow, D., Verrel, J., and Lindenberger, U. (2015). The influence of cognitive load and walking speed on gait regularity in children and young adults. Gait Posture 41, 258-262. doi: 10.1016/j.gaitpost.2014.10.013

Schott, N., and Klotzbier, T. J. (2018). Profiles of cognitive-motor interference during walking in children: does the motor or the cognitive task matter? Front. Psychol. 9:947. doi: 10.3389/fpsyg.2018.00947

Shumway-Cook, A., and Woollacott, M. (2012). Motor control: Translating research into clinical practice. 4th Edn. Baltimore, MD: Wolters Kluwer Health/Lippincott Williams \& Wilkins.

Sutherland, D. (1997). The development of mature gait. Gait Posture 6, 163-170. doi: 10.1016/S0966-6362(97)00029-5

Swanson, H. L. (1999). What develops in working memory? A life span perspective. Dev. Psychol. 35, 986-1000. doi: 10.1037/0012-1649.35.4.986

Thomason, M. E., Race, E., Burrows, B., Whitfield-Gabrieli, S., Glover, G. H., and Gabrieli, J. D. (2009). Development of spatial and verbal working memory capacity in the human brain. J. Cogn. Neurosci. 21, 316-332. doi: 10.1162/jocn.2008.21028

Vygotsky, L. S. (1934). Thought and language. Cambridge, MA: MIT Press.

Wickens, C. D. (1991). "Processing resources and attention" in Multiple-task performance. ed. D. Damos (London: Taylor \& Francis), 3-34.

Willis, C. S., and Gathercole, S. E. (2001). Phonological short-term memory contributions to sentence processing in young children. Memory 9, 349-363. doi: $10.1080 / 09658210143000155$ 
Yogev-Seligmann, G., Hausdorff, J. M., and Giladi, N. (2008). The role of executive function and attention in gait. Mov. Disord. 23, 329-342. doi: $10.1002 /$ mds. 21720

Conflict of Interest Statement: The authors declare that the research was conducted in the absence of any commercial or financial relationships that could be construed as a potential conflict of interest.
Copyright (c) 2019 Rabaglietti, De Lorenzo and Brustio. This is an open-access article distributed under the terms of the Creative Commons Attribution License (CC BY). The use, distribution or reproduction in other forums is permitted, provided the original author(s) and the copyright owner(s) are credited and that the original publication in this journal is cited, in accordance with accepted academic practice. No use, distribution or reproduction is permitted which does not comply with these terms. 